\title{
Papel de los estímulos ambientales asociados a la droga en el desarrollo de tolerancia cruzada a los efectos de taquicardia de la nicotina y el alcohol en humanos
}

\author{
Role of drug-associated environmental stimuli in \\ the development of cross-tolerance to the tachycardic \\ effects of nicotine and alcohol in humans
}

\author{
Rosa Isela Ruiz-García*, laura Nayeli Cedillo*, Juan Carlos Jiménez*, Florencio Miranda*.
}

* Facultad de Estudios Superiores Iztacala. UNAM. México.

\section{Resumen}

De acuerdo con el modelo de condicionamiento pavloviano, las claves ambientales asociadas a la droga modulan la tolerancia a las drogas. Este estudio evaluó la contribución de las claves asociadas a la droga en el desarrollo de tolerancia cruzada a los efectos taquicárdicos de la nicotina de tabaco y el alcohol en sujetos humanos. En este experimento participaron cuarenta estudiantes universitarios. Cada estudiante fue asignado aleatoriamente a una de dos condiciones experimentales. Veinte estudiantes fumaron cigarros con nicotina en el Contexto A y placebo en el Contexto B y veinte estudiantes fumaron cigarros con nicotina en el Contexto B y placebo en el Contexto A. La prueba de tolerancia cruzada fue llevada a cabo dividiendo a los participantes de cada condición en dos subgrupos $(n=10)$, cada subgrupo consumió alcohol en cada uno de los contextos (A y B). Los resultados de este experimento muestran que la tolerancia cruzada entre nicotina y alcohol se presentó únicamente cuando la prueba de tolerancia cruzada se realizó en el mismo contexto donde se desarrolló la tolerancia a la nicotina del tabaco. Estos resultados concuerdan con la hipótesis de que los estímulos ambientales asociados a la droga juegan un papel modulador en el desarrollo de la tolerancia cruzada entre la nicotina del tabaco y el alcohol.

Palabras clave: Tolerancia cruzada; condicionamiento clásico; tabaco; nicotina; alcohol; frecuencia cardíaca.

\begin{abstract}
According to the Pavlovian conditioning model, drug tolerance is modulated by drug-associated environmental cues. This study evaluated the contribution of drug-associated cues in the development of cross-tolerance to the tachycardic effects of nicotine from tobacco and alcohol in human subjects. Forty undergraduate students were recruited for this experiment, and each student was randomly assigned to one of two experimental conditions. Twenty students smoked nicotine-containing cigarettes in context $\mathrm{A}$ and placebo cigarettes in context $\mathrm{B}$, and twenty students smoked nicotine-containing cigarettes in context $\mathrm{B}$ and placebo cigarettes in context $\mathrm{A}$. A cross-tolerance test was carried out by dividing the subjects in each condition into two subgroups $(\mathrm{n}=10)$. Each subgroup consumed alcohol in both contexts (A and B). The results of this experiment showed that cross-tolerance between nicotine and alcohol was exhibited only if the cross-tolerance test was carried out in the same context where tolerance had developed to the nicotine from tobacco. These results support the hypothesis that drug-associated environmental stimuli play a modulatory role in the development of cross-tolerance between nicotine from tobacco and alcohol.

Keywords: Cross-tolerance; classical conditioning; tobacco; nicotine; alcohol; heart rate response.
\end{abstract}


土

ay una fuerte relación entre consumir alcohol o etanol y fumar cigarros con nicotina. Los estudios clínicos han mostrado la mejor evidencia de esta relación y han sugerido que es común que los pacientes diagnosticados con dependencia del alcohol también tengan dependencia de tabaco/nicotina (Abburi et al., 2016; Abreu-Villaça, Manhaes, Krahe, Filgueiras y Ribeiro-Carvalho, 2017; Drobes, 2002; Funk, Marinelli y Lê, 2006; Oliver et al., 2013). De hecho, se estima que es frecuente que entre el $80-90 \%$ de las personas con alcoholismo también fuman cigarros con nicotina (Taslim, Soderstrom y Saeed, 2011). Igualmente, el consumo de alcohol es más elevado en fumadores que en no fumadores (Abburi et al., 2016; Oliver et al., 2013) y parece ser que el consumo de ambos nicotina de tabaco y alcohol puede aumentar o reforzar los efectos de cada sustancia (Chi y De Wit, 2003; Enggasser y Wit, 2001; Oliver et al., 2013). Un estudio con sujetos humanos mostró que la interacción de los efectos farmacológicos de la nicotina y bajas dosis de alcohol juega un papel importante en la motivación por consumir ambas sustancias, y este fenómeno contribuye a desarrollar el refuerzo cruzado y la tolerancia cruzada, además de la dependencia de ambas sustancias (Oliver et al., 2013).

En este sentido, varios estudios han aportado evidencia que la nicotina y el alcohol pueden interactuar de varias maneras. Algunos estudios que han explorado los efectos conductuales a largo plazo de la nicotina y el alcohol han mostrado que el uso crónico de una sustancia induce tolerancia a los efectos conductuales y fisiológicos de la otra, lo que aumenta el potencial de coabuso (Taslim et al., 2011). Otro estudio en el que se sometió a ratones a un tratamiento crónico de diferentes dosis de alcohol encontró que los ratones también desarrollaron tolerancia a los efectos hipotérmicos de una dosis aguda de nicotina (Majchrzak y Dilsaver, 1992). Estudios in vitro también han mostrado que la exposición crónica al alcohol disminuyó la liberación de dopamina (DA) inducida por la nicotina (Dohrman y Reiter, 2003).

La tolerancia cruzada ocurre cuando el desarrollo de tolerancia a una sustancia genera tolerancia a una segunda sustancia, y el desarrollo de una tolerancia cruzada entre la nicotina y el alcohol podría explicar el aumento del consumo de ambas sustancias y contribuir al coabuso. A pesar de la dificultad en valorar el desarrollo de la tolerancia cruzada entre la nicotina y el alcohol en sujetos humanos, ambas sustancias se usan y abusan con frecuencia y la tolerancia cruzada entre ambas está ampliamente documentada en modelos animales. Por ejemplo, la nicotina y el alcohol producen efectos hipertérmicos y taquicárdicos. Estudios con ratones han mostrado que la administración crónica de alcohol en ratones mediante una dieta líquida, que induce tolerancia a varios efectos del alcohol, también produce una tolerancia cruzada a los efectos hipertérmicos y taquicárdicos de la nicotina (Collins, Burch, De Fiebre y Marks, 1988).

La perspectiva neurobiológica de la tolerancia cruzada entre la nicotina y el alcohol ha sugerido al menos cuatro posibles mecanismos en base a los sitios de acción superpuestos para el tabaco y el alcohol o las vías neuronales donde ambas sustancias ejercen sus efectos de recompensa, en particular en el sistema mesolímbico dopaminérgico. El primer mecanismo sugerido es que ambas sustancias pueden modular el receptor de acetilcolina tipo nicotínicos. Está claro que los receptores de acetilcolina nicotínicos son el sitio principal donde actúa la nicotina (Adams, 2017); sin embargo, se ha sugerido que el etanol puede interactuar directa o indirectamente con estos receptores, quizás porque el etanol estabiliza la apertura del canal. Algunos autores han sugerido que el etanol y la nicotina podrían desensibilizar los receptores nicotínicos en el sistema nervioso central (SNC) (Adams, 2017; Collins et al., 1988). El segundo mecanismo superpuesto entre la nicotina y el etanol es su capacidad para aumentar la liberación de los neurotransmisores, como DA, serotonina, glutamato y GABA. Una tercera explicación neurobiológica de la interacción entre la nicotina y el alcohol es su capacidad de sensibilizar los sistemas de factor liberador de corticotropina, un componente del sistema de estrés (Abreu-Villaça et al., 2017; Funk et al., 2006). El cuarto mecanismo en el cual interactúan el alcohol y la nicotina es la activación del sistema cerebral de recompensa. Ambas sustancias aumentan la actividad del sistema mesolímbico dopaminérgico y generan una interacción funcional entre la nicotina y el etanol (Adams, 2017). Por último, es importante tener en cuenta que hay factores genéticos comunes asociados a ambas sustancias (De Fiebre y Collins, 1993; Madden, Bucholz, Martin y Heath, 2000). La tolerancia cruzada entre la nicotina y el alcohol claramente puede tener una explicación neurobiológica. Sin embargo, este enfoque no explica la influencia de los estímulos ambientales reportada en estudios anteriores.

Hay varias maneras en las cuales los estímulos ambientales pueden afectar la conducta de las personas, por ejemplo, en la discriminación visual de una bebida alcohólica o su contenido alcohólico (Sillero-Rejon, Maynard e Ibañez-Zapata, 2020), o en la variación de los niveles de control inhibitorio en los entornos en la vida real relacionados con el alcohol, donde estímulos visuales y auditivos asociados con el alcohol rodean a las personas y pueden afectar su capacidad para controlar su consumo (Qureshi et al., 2021). Ha habido enfoques desde otras perspectivas de los mecanismos conductuales implicados en la tolerancia de las sustancias. El modelo de condicionamiento pavloviano de la tolerancia a las sustancias, propuesto por primera vez por Siegel (1977), podría explicar la tolerancia cruzada entre la nicotina y el alcohol. Este modelo sugiere que los estímulos ambientales asociados a la administración de una 
sustancia adquieren la función del estímulo condicionado (EC) y la estimulación farmacológica actúa como el estímulo incondicionado (EI). El EC asume el papel central en el desarrollo de la tolerancia porque genera tolerancia al elicitar una respuesta condicionada compensatoria (RCC) que atenúa los efectos incondicionales de una sustancia (González, Navarro, Miguez, Betancourt y Laborda, 2016; Ruiz, Vila y Miranda, 2010; Vila, Ruiz, Trejo y Miranda, 2013). En la ausencia del EC, la RCC no ocurre y, por tanto, los efectos de la sustancia no se reducen (González et al., 2016; Ruiz et al., 2010; Siegel, 1979; Siegel, Baptista, Kim, McDonald y Weise-Kelly, 2000; Siegel y Ramos, 2002; Vila et al., 2013). Una consecuencia lógica del modelo de Siegel es que la administración de una segunda sustancia durante la prueba de tolerancia en la presencia del estímulo asociado con la primera sustancia provocaría una RCC que atenuaría los efectos incondicionales de la segunda sustancia, produciéndose así la tolerancia cruzada.

Conforme al mecanismo anterior, algunos estudios han informado que los procesos de condicionamiento pavloviano podrían estar involucrados en la tolerancia cruzada a varias sustancias (Cappell, Roach y Poulos, 1981). A pesar de la realización de varios experimentos de tolerancia cruzada con animales de laboratorio (Cappell et al., 1981), todavía queda por demostrar si estos procesos de condicionamiento también regulan la tolerancia cruzada en personas al consumir dos sustancias lícitas, i.e., la nicotina y el alcohol (Oliver et al., 2013). La investigación de estos procesos implícitos en la tolerancia cruzada podría ser útil para desarrollar un mejor tratamiento para la adicción a estas sustancias. Por tanto, este estudio evaluó la participación de los procesos de condicionamiento pavloviano en la tolerancia cruzada a los efectos taquicárdicos de la nicotina de tabaco y el alcohol en sujetos humanos.

\section{Métodos}

\section{Participantes}

La muestra estuvo compuesta de cuarenta estudiantes universitarios de la Facultad de Estudios Superiores Iztacala, UNAM (23 hombres y 17 mujeres) con una edad media de 21 años. Los criterios de inclusión fueron que fumaran entre 5 y 7 cigarros diarios y bebieran alcohol entre 1 y 2 veces al mes. Identificamos a los participantes con poca dependencia de la nicotina y el alcohol (máximo de 4 puntos en el test de Fagerström; máximo de 5 puntos en el test AUDIT). Los participantes tenían un peso medio de entre 60 y $70 \mathrm{~kg}$ y altura media de entre 1,60 y 1,70 m. Los criterios de exclusión fueron la presencia de problemas de salud o la toma de fármacos recetados al inicio o durante el estudio. Se le explicó a cada sujeto el protocolo experimental y se les informó de las normas y los principios éticos de los estudios con humanos según el código ético de la Psicología (Sociedad Mexicana de Psicología, 2009; American Psy- chological Association, 2010). Los estudiantes participaron voluntariamente y dieron su consentimiento informado antes de empezar el experimento. Tenían la libertad para abandonar la tarea en cualquier momento del proceso, aunque no se dio el caso. Se les pidió que no usasen cigarros durante tres días antes y durante el experimento. Este período no provocó síntomas de abstinencia ni cambios en la respuesta cardiovascular evaluada, por lo que permitió una línea basal estable en todos los sujetos.

\section{Materiales}

Se registró las frecuencias cardíacas de los sujetos con fotopletismografia (HR / BVP IOIT: Thought Technology LTD, Quebec, Canadá). Además, se utilizó una grabadora de sonido AIWA 130, un CD de música reggae e instrucciones grabadas para relajar a los sujetos.

\section{Sustancias y placebo}

Utilizamos dos tipos de sustancias: cigarros con nicotina (Marlboro, con aproximadamente $0,9 \mathrm{mg} /$ nicotina/cigarro) y alcohol (vodka Absolut, con $40^{\circ}$ de alcohol puro). Se pidió a los sujetos que bebieran alcohol en una mezcla de vodka $(0,65 \mathrm{~g} / \mathrm{kg})$ en $100 \mathrm{ml}$ de zumo de naranja. La fórmula para calcular la cantidad de alcohol fue la siguiente:

Gramos de alcohol $=\frac{\text { volumen }(\text { en cc }) \times \text { graduación } \times 0,8}{100}$

Como placebo, se eligieron cigarros sin nicotina ni cualquier sustancia que pudiera causar un efecto en el sistema cardiovascular: cigarros de lechuga Reef LiveTM.

\section{Situación experimental}

Realizamos las sesiones en dos contextos. El Contexto A y B se crearon en una sala iluminada por dos lámparas de luz blanca, con una mesa $(1,7 \mathrm{~m}$ x $0,90 \mathrm{~m})$, una silla y un extractor de aire que permaneció en funcionamiento durante todas las sesiones. Había una mesa adicional en la sala donde se colocaron el ordenador y la grabadora de sonido. La intensidad de la luz y la música se usaron para crear diferencias entre los contextos. Contexto A incluía una luz de baja intensidad $(30 \mathrm{~W})$ y música. Contexto B estaba iluminado por lámparas de 100 W, 100 V y no tenía música.

\section{Procedimiento}

Los sujetos fueron asignados aleatoriamente a una de ambas condiciones experimentales, Contexto A o Contexto $\mathrm{B}$, de forma contrabalanceada. Veinte sujetos fumaron cigarros con nicotina en el Contexto A y cigarros placebo en el Contexto B; los 20 sujetos restantes fumaron cigarros con nicotina en el Contexto B y cigarros placebo en el Contexto A. Cada sujeto participó individualmente en una sesión de una hora. Uno de los investigadores comenzó la sesión informando a cada sujeto sobre el procedimiento para fumar. Después de responder a preguntas, otro inves- 
tigador registró la frecuencia cardíaca de los sujetos. Seguidamente, se pidió a los sujetos que escucharan las instrucciones grabadas y se relajaran durante 10 minutos, período tras el cual se registró su frecuencia cardíaca otra vez.

Fase de adquisición. Los ensayos de nicotina o placebo se presentaron en un orden contrabalanceado. Esta fase implicó cuatro ensayos por sesión. Cuando la sesión consistió en fumar cigarros con nicotina en el Contexto A, los ensayos se realizaron como sigue. Tres minutos antes de que los sujetos fumaran la primera bocanada, se realizó un cambio en la intensidad de la luz ambiental de la habitación (de normal a baja intensidad) y se activó la música. Cada ensayo consistió en fumar cuatro inhalaciones con un intervalo de 20 segundos entre inhalaciones. Cada bocanada consistía en aspirar el humo del cigarro por vía oral durante dos segundos y mantener el humo en los pulmones durante dos segundos. La frecuencia cardíaca se registraba cinco minutos tras la última bocanada. Durante el intervalo entre las pruebas, se cambió la intensidad de la luz (de $30 \mathrm{~W}$ a 100 W) y se apagó la música. Al fumar cigarros con nicotina en el Contexto B, los ensayos fueron idénticos respecto de la administración de la sustancia, pero sin cambios en la luz ambiental o la música.

El placebo (cigarros de lechuga) se administró en las mismas condiciones ambientales, es decir, en el Contexto A y en el Contexto B.

Fase de prueba de tolerancia. La prueba de tolerancia se realizó en ambos Contextos A y B. El orden de presentación de los contextos fue contrabalanceado. La prueba de tolerancia se realizó cinco minutos después del último ensayo de adquisición. En ambos contextos, a todos los sujetos se les permitió fumar cigarros con nicotina; las instrucciones para fumar fueron idénticas a las descritas en la fase anterior. La frecuencia cardíaca se registraba cinco minutos tras la última bocanada.

Fase de prueba de tolerancia cruzada. Se realizó una prueba de tolerancia cruzada como sigue. Un investigador dio instrucciones para consumir la bebida alcohólica en aproximadamente 3 minutos. Los sujetos bebieron alcohol en los dos contextos diferentes (A y B) contrabalanceando el orden de presentación del contexto. Cinco minutos después de consumir la bebida, un investigador registró su frecuencia cardíaca.

Fase de readquisición. Esta fase fue idéntica a la primera fase de adquisición; se realizó para eliminar la influencia que podría tener la evaluación previa y estabilizar la tolerancia para la prueba posterior.

Fase de prueba RCC. Se realizó la prueba RCC cinco minutos después del último ensayo de readquisición. Para la prueba RCC, a todos los sujetos se les permitió fumar cigarros que contenían lechuga; las instrucciones para fumar fueron idénticas a la fase anterior; cada sujeto consumió placebo (cigarros de lechuga) en cada contexto.

La Tabla 1 muestra un resumen del procedimiento.
Tabla 1. Desarrollo de la tolerancia cruzada.

\begin{tabular}{ccccc}
\hline Adquisición & $\begin{array}{c}\text { Prueba de } \\
\text { tolerancia }\end{array}$ & $\begin{array}{c}\text { Prueba de } \\
\text { tolerancia } \\
\text { cruzada }\end{array}$ & Readquisición & $\begin{array}{c}\text { Prueba de } \\
\text { RCC }\end{array}$ \\
\hline A: NIC & A: NIC & A: ALC & A: NIC & A: $P$ \\
B: $P$ & B: NIC & B: ALC & B: $P$ & B: $P$ \\
A: $P$ & A: NIC & A: ALC & A: $P$ & A: $P$ \\
B: NIC & B: NIC & B: ALC & B: NIC & B: $P$ \\
\hline
\end{tabular}

Nota. $\mathrm{A}=$ Contexto $\mathrm{A}, \mathrm{B}=$ Contexto $\mathrm{B}, \mathrm{NIC}=$ Nicotina $, \mathrm{ALC}=\mathrm{Alcohol}, \mathrm{P}=$ Placebo

\section{Análisis estadístico}

La frecuencia cardíaca de cada sujeto se registró en la línea basal, durante el entrenamiento y durante la prueba de tolerancia cruzada. Los datos basales se analizaron mediante un ANOVA de dos vías, con la condición (condiciones de nicotina y placebo) como primer factor y la línea base (antes y después de la relajación) como segundo factor. Durante la fase de entrenamiento, los datos se analizaron mediante un ANOVA de dos vías con medidas repetidas, con las condiciones de nicotina y placebo como primer factor y el número de prueba como segundo factor. Durante la prueba de tolerancia cruzada, los datos se analizaron con la prueba $t$ de Student. Los datos de la fase de readquisición se analizaron mediante un ANOVA de dos vías con medidas repetidas, con las condiciones de nicotina y placebo como primer factor y el número de prueba como segundo factor. Los datos se analizaron mediante la prueba $t$ de Student para la prueba de la RCC. Cuando los ANOVA fueron significativos, se realizaron comparaciones múltiples utilizando la prueba de Tukey. El nivel de rechazo para el error tipo I fue de 0,05 en todas las pruebas.

\section{Resultados}

\section{Fase de adquisición}

Los resultados de la línea basal (antes y después de la relajación) se muestran en el lado izquierdo de la Figura 1-A. El ANOVA de dos vías indicó que no hubo diferencias entre la condición de nicotina (84,8 latidos por minuto) y la condición de placebo (83,4 latidos por minuto) antes de la relajación. A pesar de la disminución significativa en la frecuencia cardíaca después de la relajación en ambas condiciones, no hubo diferencias entre las condiciones de nicotina (72,8 latidos por minuto) y placebo (70,9 latidos por minuto) después de la relajación $(\mathrm{F}[1,39]=, 357, \mathrm{p}$ $>, 05 ; \mathrm{F}[1,39]=107,601, \mathrm{p}<, 05$; respectivamente). Además, la prueba reveló que no hubo diferencias entre las condiciones antes y después basadas en las condiciones de nicotina y placebo (es decir, no hubo interacción) (F [1, $39]=1,040, p>, 05)$. En resumen, tras la relajación, todos los sujetos comenzaron el experimento con una frecuencia 


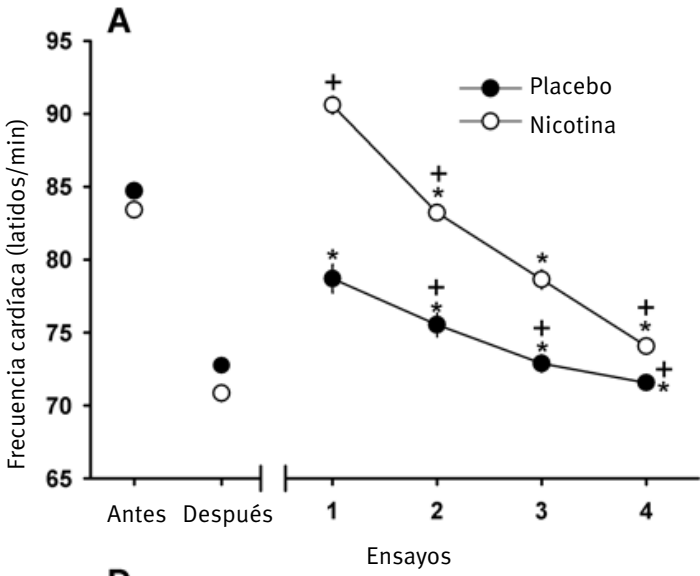

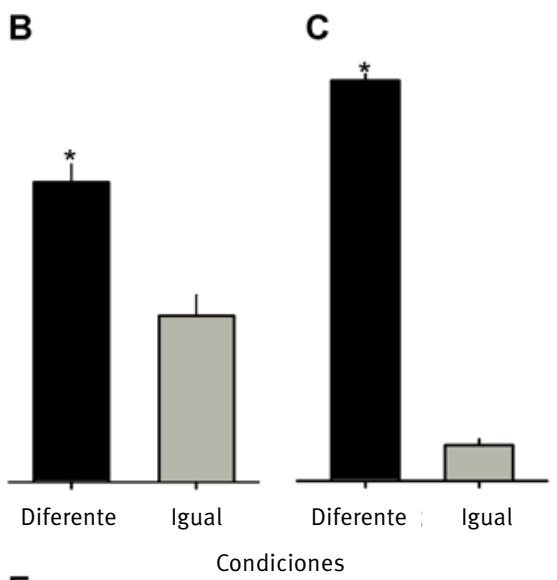

E

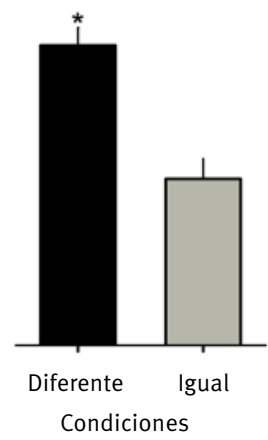

Nota. Los resultados se representan como la media de la frecuencia cardíaca de los sujetos en \pm SEM. A. Desarrollo de tolerancia a la nicotina. Los primeros dos puntos representan el antes y después del período de relajación. También se muestra el desarrollo de la tolerancia condicionada a los efectos taquicárdicos; los círculos no sombreados muestran los efectos después de fumar varios cigarros con nicotina, y los círculos sombreados muestran el efecto obtenido tras fumar cigarros placebo. El asterisco $\left(^{*}\right)$ indica diferencias significativas respecto del primer ensayo con nicotina. La cruz (+) indica diferencias significativas respecto del primer ensayo con placebo. B. Prueba de tolerancia. Frecuencia cardíaca de los sujetos que fumaron nicotina en el contexto Diferente o Mismo. El asterisco $(*)$ indica diferencias significativas respecto del Mismo contexto. C. Prueba de tolerancia cruzada. Resultados obtenidos cuando los sujetos bebieron alcohol en los contextos Diferente y Mismo. El asterisco ${ }^{*}$ ) indica diferencias significativas respecto del Mismo contexto. D. Fase de readquisición. Muestra la readquisición de la tolerancia; los círculos no sombreados muestran los efectos de la nicotina, y los círculos sombreados muestran el efecto obtenido tras fumar cigarros placebo. E. Prueba RCC. Resultados obtenidos cuando los sujetos fumaron cigarros placebo en los contextos Diferente y Mismo. El asterisco $\left(^{\star}\right)$ indica diferencias significativas respecto del Mismo contexto.

Figura 1. Papel de las señales asociadas con la sustancia en el desarrollo de la tolerancia cruzada ante los efectos taquicárdicos de la nicotina de tabaco y alcohol.

cardíaca constante de aproximadamente 70-72 latidos por minuto.

Los cambios en la tasa cardiaca a través de los cuatro ensayos de adquisición de tolerancia se muestran en la parte derecha de la Figura 1A. Los datos mostraron que el efecto inicial de la nicotina del tabaco fue incrementar la tasa cardiaca. Un ANOVA de medidas repetidas de dos factores, con los grupos como primer factor y el número de ensayos como segundo factor, indicaron que existen diferencias significativas entre la condición nicotina y la condición placebo $(F[1,38]=19,204, \mathrm{p}<0,01)$, entre ensayos $(F[5,90]$ $=36,779, \mathrm{p}<0,01)$ y en la interacción de grupo-ensayo $(F[5$, $90]=10,541, \mathrm{p}<0,01)$. Como puede observarse, el efecto sobre la tasa cardiaca disminuyó en función del número de exposiciones a la droga. El análisis estadístico realizado con la prueba Tukey señaló que, en el caso de la condición nicotina, existe una disminución significativa a partir del ensayo $2(\mathrm{p}<0,05)$. Por otra parte, en la condición placebo, aunque se observa un aumento en el ensayo 1 con respecto a la línea base, la tasa cardiaca promedio es significativamente menor con respecto a la observada en el ensayo 1 de la condición placebo. La respuesta de la tasa cardiaca fue significativamente menor que la observada en el ensayo 1 de la condición nicotina $(\mathrm{p}<0,05)$.

En la condición de placebo, los ensayos no presentaron diferencias significativas entre ellos, comparado con el ensayo 4 de la condición de nicotina o comparado con después de la relajación.

\section{Fase de prueba de tolerancia}

Los resultados de la fase de prueba de tolerancia mostraron que cuando los sujetos fumaron cigarros con nicoti- 
na en el contexto asociado con el consumo de la sustancia, mostraron una disminución de frecuencia cardíaca (barras señaladas «Igual» en la Figura 1B), mientras que cuando los sujetos fumaron cigarros con nicotina en ausencia de los estímulos ambientales asociados con la sustancia, su frecuencia cardíaca aumentó (barra señalada «Diferente» en la Figura 1B). La prueba $t$ de Student relacionada confirmó una diferencia significativa entre las respuestas en estas dos condiciones $(\mathrm{t}[38]=3,707, \mathrm{p}<, 05)$.

\section{Fase de prueba de tolerancia cruzada}

La Figura 1C muestra los resultados de la prueba de tolerancia cruzada. Cuando los sujetos bebieron alcohol en el ambiente asociado con fumar cigarros con nicotina, su frecuencia cardíaca disminuyó (barra señalada «Igual» en la Figura 1C), mientras que cuando bebieron alcohol en la ausencia de estímulos ambientales asociados con fumar cigarros con nicotina, su frecuencia cardíaca aumentó (barra señalada «Diferente» en la Figura 1C). La prueba $t$ de Student relacionada indicó una diferencia entre las respuestas en estas dos condiciones ( $[38]=15,036, \mathrm{p}<, 05)$.

\section{Fase de readquisición}

La Figura 1D muestra los cambios en la frecuencia cardíaca en los cuatro ensayos de readquisición de tolerancia. Los datos de esta fase mostraron que el efecto inicial de fumar cigarros con nicotina no aumentó la frecuencia cardíaca, comparado con la respuesta basal. Como se puede observar, este efecto se mantuvo a lo largo de todos los ensayos y se hizo menos pronunciado, comparado con los ensayos en la condición de placebo. Los ANOVA de dos vías con medidas repetidas indicaron que no hubo diferencias significativas entre los grupos $(F[1,38]=2,01, p>, 05)$. Los resultados también mostraron que no hubo diferencias significativas entre ensayos $(\mathrm{F}[5,70]=, 248, \mathrm{p}>, 05)$ y no hubo interacción condición-ensayos $(\mathrm{F}[3,114]=, 391, \mathrm{p}>, 05)$.

\section{Fase de prueba RCC}

Los resultados de la prueba RCC en la fase de readquisición mostraron que cuando los sujetos fumaron cigarros con lechuga en el contexto asociado con el consumo de la sustancia, su frecuencia cardíaca disminuyó (barra señalada «Igual» en la Figura 1E); mientras que, cuando los sujetos fumaron cigarros con lechuga en la ausencia de los estímulos ambientales asociados con la sustancia, su frecuencia cardíaca aumentó (barra señalada «Diferente» en la Figura 1E). Las pruebas $t$ de Student relacionadas confirmaron una diferencia significativa entre las respuestas en las dos condiciones $(\mathrm{t}[19]=6,688, \mathrm{p}<, 05)$.

\section{Discusión}

Este estudio tuvo como propósito evaluar la contribución de las señales relacionadas con las sustancias en el de- sarrollo de la tolerancia cruzada a los efectos taquicárdicos de la nicotina de tabaco y el alcohol en sujetos humanos. También se evaluó la expresión de la RCC como un posible mecanismo subyacente de la tolerancia cruzada entre la nicotina y el alcohol en los efectos taquicárdicos. Encontramos que el desarrollo de la tolerancia a los efectos taquicárdicos de la nicotina puede modularse por las señales ambientales asociados a su consumo. Los datos también mostraron tolerancia cruzada entre la nicotina y el alcohol en las frecuencias cardíacas, y este efecto solo se observó si la prueba de tolerancia cruzada se realizó en el mismo contexto en el que se desarrolló la tolerancia a la primera sustancia; cuando la prueba de tolerancia cruzada se llevó a cabo en un contexto diferente, la tolerancia cruzada se invirtió y la frecuencia cardíaca aumentó. También se observó evidencia de una RCC cuando los sujetos consumieron placebo en presencia de estímulos ambientales asociados con el consumo de nicotina y su frecuencia cardíaca disminuyó, comparado con los sujetos que consumieron placebo en ausencia de estos estímulos; los resultados sugieren que una RCC podría ser el mecanismo subyacente de la tolerancia cruzada.

Los resultados conductuales descritos anteriormente son coherentes con los de estudios existentes que demuestran la influencia de la especificidad del contexto de la tolerancia en diferentes procedimientos con ratas y con diferentes sustancias, incluida la nicotina (Field y Duka, 2001; McDermut y Haaga, 1998; Mucha, Pauli y Angrilli, 1998; Naqvi y Bechara, 2006) y el alcohol (Duncan, Alici y Woodward, 2000; Le, Poulos y Cappell, 1979; White, Roberts y Best, 2002).

Con respecto al papel modulador del contexto ambiental en la tolerancia cruzada, nuestros resultados son la primera evidencia que muestra la contribución de las señales asociadas con la sustancia en el desarrollo de la tolerancia cruzada entre los efectos de la nicotina del tabaco y los efectos del alcohol en sujetos humanos. Sugieren que la tolerancia que se desarrolla a los efectos de una sustancia particular en un contexto específico puede contribuir a la expresión de tolerancia a los efectos de una segunda sustancia que los sujetos no han consumido previamente si la segunda sustancia se consume en el mismo ambiente donde se consumió la primera. Por tanto, podemos sugerir que los procesos de condicionamiento pavloviano que contribuyeron a la expresión de la tolerancia cruzada entre la nicotina y el alcohol correspondían a los efectos descritos en los estudios anteriores realizados con diferentes procedimientos con animales y podrían utilizarse como evidencia en relación con el conocimiento de los fenómenos que contribuyen a desarrollar y mantener la adicción a las sustancias, dado que el desarrollo de la tolerancia cruzada puede ser un componente importante en la progresión o el aumento del consumo y el desarrollo de la dependencia de las sustancias de abuso (Cappell et al., 1981; Car- 
mona-Perera, Sumarroca-Hernández, Santolaria-Rossell, Pérez-García y Reyes del Paso, 2019; Collins et al., 1988; De Fiebre y Collins, 1993; Oliver et al., 2013).

Una explicación inicial de los resultados de nuestro estudio implica evaluar los mecanismos que subyacen el desarrollo de la adicción y dependencia a la nicotina y al alcohol, que han sido ampliamente evaluados (Little, 2000). Los mecanismos neurobiológicos constituyen un primer conjunto de factores que han servido como explicación no solo de estos fenómenos sino también de la tolerancia cruzada entre la nicotina y el alcohol. En este caso, la explicación se centró en las acciones indirectas de estas sustancias sobre el sistema de recompensas. La nicotina aumenta las concentraciones de DA en el núcleo accumbens (NAcc) por tres mecanismos diferentes (De Kloet, Mansvelder y De Vries, 2015): activación de las neuronas del área tegmental ventral (ATV) a través de receptores nicotínicos con subunidades $\alpha 4, \alpha 6, \alpha 7$ y $\beta 2$; activación de neuronas DAérgicas por activación colinérgica del núcleo pedunculopontino; e inhibición de interneuronas GABAérgicas en el VTA por desensibilización de receptores nicotínicos con subunidades $\beta 2$. Por otro lado, el alcohol también aumenta las concentraciones de DA y actúa sobre los receptores GABAA en las interneuronas GABAérgicas en el ATV. La activación de GABAA disminuye la liberación de GABA en el ATV y, por tanto, aumenta la liberación de DA en el NAcc (Leggio, Kenna y Swift, 2008). Además, se sabe que el alcohol y la nicotina pueden producir efectos diferenciales en distintas variables conductuales y fisiológicas que podrían ser iguales, según la dosis de sustancia consumida, e incluso se ha sugerido que ambas sustancias comparten al menos un componente genético que podría producir una predisposición al uso o abuso de las mismas (Funk et al., 2006). Basado en la similitud en los mecanismos, se ha propuesto que la estimulación farmacológica de sitios específicos por parte de la nicotina genera cambios plásticos en las neuronas. Dado que estos cambios no desaparecen y son el mismo sitio de acción de la segunda sustancia, cuando se consume alcohol por primera vez, se produce el fenómeno de tolerancia, incluso en la ausencia de experiencia previa. Esta explicación farmacológica parece aclarar el fenómeno de tolerancia cruzada; sin embargo, no explica la especificidad ambiental de la tolerancia cruzada. Es decir, no explica por qué la tolerancia cruzada se produjo sólo en el contexto donde la tolerancia se había desarrollado y no en otro. La tolerancia cruzada, por tanto, puede estudiarse como un fenómeno farmacológico o como un fenómeno de aprendizaje; sin embargo, las teorías farmacológicas son silenciosas respecto del papel de los estímulos ambientales asociados con la administración de una sustancia en el desarrollo de tolerancia cruzada o con respecto al valor de incentivo de una sustancia. Por tanto, comprender cómo el desarrollo de la tolerancia a una sustancia puede modular la tole- rancia a otra segunda sustancia requiere una comprensión tanto de principios de aprendizaje como de principios farmacológicos.

Otro factor que podría contribuir a explicar el desarrollo de la tolerancia cruzada es el papel de los estímulos ambientales asociados con la administración de una sustancia. El modelo de condicionamiento de tolerancia a drogas propuesto por Siegel (1977) predice que el desarrollo de tolerancia está influenciado por variables ambientales, particularmente el historial de asociación de estímulos ambientales con la administración de una sustancia. Por tanto, el desarrollo de la tolerancia ocurre porque los estímulos condicionados causan una RC opuesta a los efectos de la sustancia, y esta RC antagonista atenúa sus efectos incondicionales (Dafters y Anderson, 1982; Duncan et al., 2000; Newlin, 1986; Siegel, 1977, 1979).

Además de la especificidad contextual que proporciona una base empírica para la participación del condicionamiento pavloviano en la tolerancia, el modelo indica que la producción de una RCC es fundamental para explicar la tolerancia. La RCC suele evidenciarse en sujetos que se han sometido a un proceso de adquisición y desarrollo de tolerancia a una droga, y en una fase posterior, se administra un placebo en presencia de los estímulos ambientales asociados con la administración crónica de la droga (Newlin, 1986; Ruiz et al., 2010). Un ejemplo es un estudio previo que hicimos en nuestro laboratorio que muestra que los estímulos ambientales son un componente importante para explicar la tolerancia; los estímulos que produjeron la RCC atenuaron los efectos incondicionales de la droga, pero la ausencia de estos estímulos no provocó la RCC; por tanto, no se observaron efectos reducidos de la droga (Ruiz et al., 2010).

De esta manera, el modelo podría sugerir una explicación adicional de la tolerancia cruzada entre la nicotina y el alcohol observada en el experimento actual. Los estímulos ambientales asociados con fumar cigarros con nicotina podrían haber producido una RCC capaz de sumarse a los efectos agudos del alcohol (Cappell et al., 1981; González et al., 2016; Ruiz et al., 2010; Vila et al., 2013). En otras palabras, los estímulos contextuales disminuyeron la frecuencia cardíaca en los sujetos en la prueba de tolerancia cruzada. Este efecto se sumó algebraicamente al aumento de la frecuencia cardíaca causada por el consumo de alcohol. Observamos como resultado una disminución en los efectos agudos del alcohol sobre la frecuencia cardíaca, comparado con los efectos observados en la ausencia de los estímulos contextuales; esto nos permite suponer que la RCC también subyace a la tolerancia cruzada observada en este experimento. De esta manera, nuestros resultados apoyan la hipótesis propuesta por varios investigadores que las señales contextuales son un componente importante para explicar la tolerancia cruzada entre la nicotina y el alcohol, asignando un papel central a los procesos aso- 
ciativos pavlovianos (Ruiz et al., 2010; Siegel, 1979, Siegel et al., 2000; Siegel y Ramos, 2002; Vila et al. 2013). Aunque estos resultados no pueden reducirse a mecanismos farmacológicos, podemos afirmar se complementan ambos mecanismos (farmacológico y de condicionamiento).

La importancia clínica de nuestros resultados radica en la pertinencia de la tolerancia cruzada condicionada en el abuso humano de la nicotina y el alcohol basada en la demostración de Siegel de que la letalidad de una dosis de droga puede verse muy influenciada por la presencia de estímulos relacionados con las drogas. En un entorno no relacionado con sustancias, la ausencia de respuestas condicionadas opuestas a las sustancias y, por tanto, la ausencia de tolerancia cruzada condicionada, resulta en un aumento en la potencia de la sustancia y en el potencial de sobredosis. Debido a las consecuencias potencialmente letales para el drogadicto activo, se necesita más investigación para determinar más exhaustivamente la incidencia de la tolerancia cruzada condicionada en el entorno natural y su grado de participación en una sobredosis accidental. A nivel aplicado, estos hallazgos son particularmente relevantes para los médicos, dado que los tratamientos para los trastornos relacionados con el consumo de sustancias han carecido de efectividad. Los resultados presentados aquí podrían ayudar a mejorar la terapia en el entorno clínico mediante la implementación de técnicas que han demostrado reducir la influencia de los estímulos ambientales asociados con el uso de una sustancia.

Otro objetivo de los programas de tratamiento es prevenir la recaída en el abuso de sustancias. Lo típico tras un período de desintoxicación es que un paciente no muestre angustia por abstinencia y no informe el deseo o craving por la droga. Por tanto, cuando los pacientes son dados de alta y regresan a los entornos donde antes utilizaban drogas, muestran angustia por abstinencia, sienten craving y sufren una recaída. Puede ser que el tratamiento no haya reducido la capacidad de los estímulos asociados a las drogas para provocar cravingy angustia por abstinencia, lo que los convierte en factores contribuyentes a la recaída

Estos resultados requieren de estudios futuros dado que una discusión completa de los mecanismos potenciales que explican el desarrollo de la tolerancia cruzada a las frecuencias cardíacas producidas por la nicotina y el alcohol requiere incorporar un análisis de los efectos simétricos de ambas sustancias utilizadas. Por tanto, sería importante evaluar si la tolerancia cruzada se desarrolla cuando se invierte el orden de presentación de las sustancias; es decir, primero administrando alcohol para desarrollar tolerancia y después nicotina para observar la tolerancia cruzada. Es más, sería importante evaluar si la tolerancia cruzada se puede observar con diferentes efectos producidos por estas sustancias.

\section{Conclusiones}

Los resultados de esta investigación mostraron que la tolerancia cruzada entre la nicotina y el alcohol fue modulada por los estímulos ambientales asociados con la administración de la primera sustancia y la RCC resultante. Nuestros resultados tienen implicaciones importantes en el estudio de los mecanismos de adicción y drogodependencia. La interacción de los factores condicionantes, como el control de los estímulos ambientales en la tolerancia y la tolerancia cruzada, podría desempeñar un papel importante en el abuso de sustancias y en la drogodependencia.

\section{Reconocimientos}

Este estudio recibió una subvención IN305420 de PAPIIT UNAM (México).

\section{Conflicto de intereses}

Los autores declaran la inexistencia de conflicto de interés.

\section{Referencias}

Abburi, Ch., Wolfman, S. L., Metz, R. A. E., Kamber, R., McGehee, D. S. y McDaid, J. (2016). Tolerance to ethanol or nicotine results in increased ethanol self-administration and long-term depression in the dorsolateral striatum. eNeuro, 112, 1-40. doi:10.1523/eneuro.0112-15.2016.

Abreu-Villaça, Y., Manhaes, A., Krahe, T., Filgueiras, C. y Ribeiro-Carvalho, A. (2017). Tobacco and alcohol use during adolescence: Interactive mechanisms in animal models. Biochemical Pharmacology, 144, 1-17. doi:10.1016/j. bcp.2017.06.113.

Adams, S. (2017). Psychopharmacology of tobacco and alcohol comorbidity: A review of current evidence. $\mathrm{Cu}$ rrent Addiction Reports, 4, 25-34. doi:10.1007/s40429-0170129-z.

American Psychological Association (2010). Ethical principles of psychologists and code of conduct, amendments. Facultad de Psicología. Universidad de Buenos Aires, Argentina.

Cappell, H., Roach, C. y Poulos, C. (1981). Pavlovian control of cross-tolerance between pentobarbital and ethanol. Psychopharmacology, 74, 54-57.

Carmona-Perera, M., Sumarroca-Hernández, X., Santolaria-Rossell, A., Pérez-García, M. y Reyes del Paso, G. A. (2019). Blunted autonomic responses to emotional stimuli in alcoholism: Relevance of impulsivity. Adicciones, 31, 221-232. doi:10.20882/adicciones.1146.

Chi, H. y De Wit, H. (2003). Mecamylamine attenuates the subjective stimulant-like effects of alcohol in social drinkers. Alcoholism: Clinical and Experimental Research, 27, 780-786. 
Collins, A., Burch, J., De Fiebre, Ch. y Marks, M. (1988). Tolerance to and cross tolerance between ethanol and nicotine. Pharmacology Biochemistry E Behavior, 29, 365373.

Dafters, R. y Anderson, G. (1982). Conditioned tolerance to the tachycardia effects of ethanol in humans. Psychopharmacology, 78, 365-367.

De Fiebre, Ch. y Collins, A. (1993). A comparison of the development of tolerance to ethanol and cross-tolerance to nicotine after chronic ethanol treatment in longand short-sleep mice. The Journal of Pharmacology and Experimental Therapeutics, 266, 1398-1406.

De Kloet, S., Mansvelder, H. y De Vries, T. (2015). Cholinergic modulation of dopamine pathways through nicotinic acetylcholine receptors. Biochemical Pharmacology, 97, 425-438. doi:10.1016/j.bcp.2015.07.014.

Dohrman, D. y Reiter, C. (2003). Ethanol modulates nicotine-induced upregulation of nAChRs. Brain Research, 975, 90-98.

Drobes, D. (2002). Concurrent alcohol and tobacco dependence. Alcohol Research and Health 26, 136-142.

Duncan, P., Alici, T. y Woodward, J. (2000). Conditioned compensatory response to ethanol as indicated by locomotor activity in rats. Behavior Pharmacology, 11, 395-402.

Enggasser, J. y Wit, H. (2001). Haloperidol reduces stimulant and reinforcing effects of ethanol in social drinkers. Alcoholism, Clinical and Experimental Research, 25, 14481456 .

Field, M. y Duka, T. (2001). Smoking expectancy mediates the conditioned responses to arbitrary smoking cues. $B e-$ havior Pharmacology, 12, 183-194.

Funk, D., Marinelli, P. W. y Lé, A. D. (2006). Biological processes underlying co-use of alcohol and nicotine: Neuronal mechanisms, cross-tolerance, and genetic factors. Alcohol Research Health, 29, 186-192.

González, V. V., Navarro, V., Migueza, G., Betancourt, R. y Laborda, M. (2016). Preventing the recovery of extinguished ethanol tolerance. Behavioural Processes, 124, 141-148. doi:10.1016/j.beproc.2016.01.004.

Le, A., Poulos, C. y Cappell, H. (1979). Conditioned tolerance to the hypothermic effect of ethyl alcohol. Science, 206, 1109-1110.

Leggio, L., Kenna, G. y Swift, R. (2008). New developments for the pharmacological treatment of alcohol withdrawal syndrome. A focus on non-benzodiazepine GABAergic medications. Progress in Neuro-Psychopharmacology E B Biological Psychiatry, 32, 1106-1117.

Little, J. H. (2000). Behavioral mechanisms underlying the link between smoking and drinking. Alcohol Research $\mathcal{E}^{\circ}$ Health, 24 , 215-224.

Madden, P. A., Bucholz, K. K., Martin, N. G. y Heath, A. C. (2000). Smoking and the genetic contribution to alcohol-dependence risk. Alcohol Research Health, 24, 209-214.
Majchrzak, M. J. y Dilsaver, S. C. (1992). Chronic treatment with ethanol alters the physiological action of nicotine. Progress in Neuro-psychopharmacology $\mathcal{E} \mathcal{F}^{2}$ Biological Psychiatry, 16, 107-115.

McDermut, W. y Haaga, D. (1998). Effect of stage of change on cue reactivity in continuing smokers. Experimental Clinical Psychopharmacology, 6, 316-324.

Mucha, R., Pauli, P. y Angrilli, A. (1998). Conditioned responses elicited by experimentally produced cues for smoking. Canadian Journal of Physiology and Pharmacology, 76, 259-268.

Naqvi, N. y Bechara, A. (2006). Skin conductance responses are elicited by the airway sensory effects of puffs from cigarettes. Journal Psychophysiology, 61, 77-86.

Newlin, D. (1986). Conditioned compensatory response to alcohol placebo in humans. Psychopharmacology, 88, 247251.

Oliver, J. A., Blank, M. D., Van, R. K., MacQueen, D. A., Brandon, T. H. y Drobes, D. (2013). Nicotine interactions with low-dose alcohol: Pharmacological influences on smoking and drinking motivation. Journal of Abnormal Psychology, 122, 1154-1165. doi:10.1037/a0034538.

Qureshi, A., Monk, R.L., Pennington, C.R., Li, X., Leatherbarrow, T. y Oulton J.R. (2021). Visual and auditory contextual cues differentially influence alcohol-related inhibitory control. Adicciones, 33, 7-18. doi:10.20882/ adicciones.1091.

Ruiz, I., Vila, J. y Miranda, F. (2010). El papel de los procesos asociativos en la manifestación de fenómenos relacionados con la adicción a las drogas. Anuario de Investigación en Adicciones, 11, 86-98.

Siegel, S. (1977). Morphine tolerance acquisition as an associative process. Journal Experimental Psychology: Animal Behavior Processes, 3, 1-13.

Siegel, S. (1979). The role of conditioning in drug tolerance and addiction (pp.143-168). En J. D. Keehn (Ed.) Psychopathology in animals: Research and treatment implications. New York: Academic Press.

Siegel, S., Baptista, M., Kim, J., McDonald, R. y Weise-Kelly, L. (2000). Pavlovian psychopharmacology: The basis of tolerance. Experimental and Clinical Psychopharmacology, 8, 276-293.

Siegel, S. y Ramos, B. (2002). Applying laboratory research: Drug anticipation and the treatment of drug addiction. Experimental and Clinical Psychopharmacology, 10, 162-183.

Sillero-Rejon, C., Maynard, O. e Ibáñez-Zapata, J. A. (2020). Visual attention to alcohol labels: an exploratory eye-tracking experiment. Adicciones, 32, 202-207. doi:10.20882/adicciones.1207.

Sociedad Mexicana de Psicología (2009). Código ético del psicólogo. México: Trillas.

Taslim, N., Soderstrom, K. y Saeed, D.M. (2011). Role of mouse cerebellar nicotinic acetylcholine receptor 
(nAChR) and subtypes in the behavioral cross-tolerance between nicotine and ethanol-induced ataxia. Behavioral Brain Research, 217, 282-292. doi:10.1016/j. bbr.2010.10.026.

Vila, J., Ruiz, I., Trejo, F. y Miranda, F. (2013). Participación de los factores de condicionamiento pavloviano en el desarrollo de tolerancia a los efectos cardiovasculares producidos por la nicotina del tabaco. Investigación Psicológica. Revista de Psicología de la Universidad de Chile, 22, 1-14. doi:10.5354/0719-0581.2014.27715.

White, A., Roberts, D. y Best, P. (2002). Context-specific tolerance to the ataxic effects of alcohol. Pharmacology Biochemistry and Behavior, 72, 107-110. 